\title{
Strategies to reduce the risk of SARS-CoV-2 importation from international travellers: modelling estimations for the United Kingdom, July 2020
}

Samuel Clifford ${ }^{1, *}$, Billy J Quilty ${ }^{1, *}$, Timothy W Russell ${ }^{1}$, Yang Liu ${ }^{1}$, Yung-Wai D Chan ${ }^{1}$, Carl A B Pearson ${ }^{1}$, Rosalind M Eggo ${ }^{1}$, Akira Endo $^{1,2}$, CMMID COVID-19 Working Group ${ }^{2}$, Stefan Flasche ${ }^{1, * *}$, W John Edmunds ${ }^{1, * *}$

1. Centre for Mathematical Modelling of Infectious Diseases, Department of Infectious Disease Epidemiology, London School of Hygiene and Tropical Medicine, London, United Kingdom

2. The members of the group are listed under Investigators

* These authors contributed equally and share first authorship

** These authors contributed equally and share last authorship

Correspondence: Samuel Clifford (sam.clifford@lshtm.ac.uku), Billy Quilty (billy.quilty@lshtm.ac.uk)

Investigators: The investigators are listed at the end of the article.

Citation style for this article:

Clifford Samuel, Quilty Billy J, Russell Timothy W, Liu Yang, Chan Yung-Wai D, Pearson Carl A B, Eggo Rosalind M, Endo Akira, CMMID COVID-19 Working Group, Flasche Stefan, Edmunds W John. Strategies to reduce the risk of SARS-CoV-2 importation from international travellers: modelling estimations for the United Kingdom, July 2020. Euro Surveill. 2021;26(39):pii=2001440. https://doi.org/10.2807/1560-7917.ES.2021.26.39.2001440

Background: To mitigate SARS-CoV-2 transmission risks from international air travellers, many countries implemented a combination of up to 14 days of selfquarantine upon arrival plus PCR testing in the early stages of the COVID-19 pandemic in 2020. Aim: To assess the effectiveness of quarantine and testing of international travellers to reduce risk of onward SARSCoV-2 transmission into a destination country in the pre-COVID-19 vaccination era. Methods: We used a simulation model of air travellers arriving in the United Kingdom from the European Union or the United States, incorporating timing of infection stages while varying quarantine duration and timing and number of PCR tests. Results: Quarantine upon arrival with a PCR test on day 7 plus a 1-day delay for results can reduce the number of infectious arriving travellers released into the community by a median $94 \%$ ( $95 \%$ uncertainty interval (UI): 89-98) compared with a no quarantine/no test scenario. This reduction is similar to that achieved by a 14-day quarantine period (median > $99 \%$; 95\% UI: 98-100). Even shorter quarantine periods can prevent a substantial amount of transmission; all strategies in which travellers spend at least 5 days (mean incubation period) in quarantine and have at least one negative test before release are highly effective (median reduction 89\%; 95\% UI: 83-95)). Conclusion: The effect of different screening strategies impacts asymptomatic and symptomatic individuals differently. The choice of an optimal quarantine and testing strategy for unvaccinated air travellers may vary based on the number of possible imported infections relative to domestic incidence.

\section{Background}

Severe acute respiratory syndrome coronavirus 2 (SARS-CoV-2), the causative agent of coronavirus disease (COVID-19), emerged in Wuhan, China in late 2019 and was rapidly disseminated globally through international air travel in the first half of 2020 [1]. In addition to non-pharmaceutical interventions (NPIs) to reduce domestic transmission, many countries implemented restrictions on incoming international travel such as mandatory quarantine, testing and travel bans, with the aim of preventing or reducing further importation and onward transmission [2].

During this early period of the COVID-19 pandemic prior to the roll-out of vaccines in late 2020, a number of countries in Europe and the Asia Pacific region implemented a mandatory quarantine upon arrival, which typically had a duration of 14 days $[2,3]$. It is expected that, by day 14 , at least $95 \%$ of all infected individuals who will become symptomatic have done so [4]. However, the median incubation period for SARSCoV-2 is ca 5 days (95\% confidence interval: 4.1 to 7.0 ) [4] and, assuming that travellers are equally likely to travel at any point in this period, a 5-day quarantine on arrival should suffice to allow more than $50 \%$ of the infected travellers to become symptomatic and be managed accordingly. Quarantine, either at home or at managed facilities [5], may lead to negative psychological effects stemming from social isolation $[6,7]$ and financial stress [8]. Hence, there is considerable interest in reducing the period of quarantine, assuming it is safe to do so. 

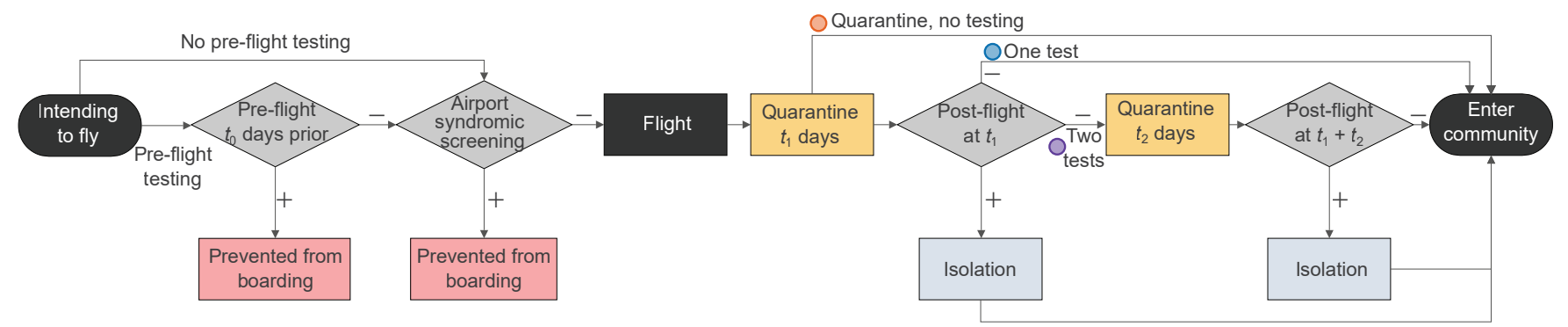

SARS-CoV-2: severe acute respiratory syndrome coronavirus 2.

Testing for SARS-CoV-2 infection (grey diamonds) may be conducted pre-flight and/or post-flight and may occur alongside quarantine periods (yellow boxes). Travellers who are found to be infected pre-flight are prevented from boarding (pink boxes); travellers found to be infected during quarantine are diverted to isolation (light blue boxes). Travellers enter the community after the required number of negative tests (regardless of infection status) or after finishing their allocated duration of quarantine or isolation. Coloured circles indicate the number of tests along that trajectory.

In addition to quarantine, several countries introduced a requirement for travellers to undergo testing for SARSCoV-2 infection with RT-PCR (hereafter PCR). Such testing is commonly performed by taking nasopharyngeal or throat swabs of individuals and analysing the resulting sample for the presence of SARS-CoV-2 RNA [9]. PCR screening may be conducted before the flight and/ or after arrival to allow detection of infected travellers. In some countries, testing is also used to reduce or eliminate quarantine for travellers without a confirmed infection. For example, in the summer of 2020, Japan allowed business travellers from designated low-risk countries to bypass the 14 -day quarantine period given a negative PCR test result upon arrival [10].

Here we investigated the effectiveness of several strategies available in the pre-vaccination era of the SARS-CoV-2 pandemic to reduce the number of arriving infectious travellers as well as the potential for transmission in the community. We assessed the impact of varying the duration of quarantine and the timing and number of PCR tests, as well as the prevalence in and travel volume from the European Union (EU) and the United States (US) to the United Kingdom (UK) as of July 2020, while also accounting for the natural history of SARS-CoV-2 infection.

\section{Methods}

\section{Travel screening trajectories}

The possible SARS-CoV-2 screening outcomes for air travellers are as follows: (i) prevented from travelling following detection of SARS-CoV-2 infection either through syndromic screening at the airport or a positive pre-flight PCR test, (ii) released after the mandatory isolation period following detection of SARS-CoV-2 infection either by a positive PCR test upon entry or a follow-up positive PCR test after a negative result upon entry, (iii) released after a second negative test during the quarantine period, and (iv) in the absence of postentry testing, travellers will be released after the mandatory quarantine period (which, in the model, may have a duration of o days) (Figure 1).

\section{Estimating the number of infected travellers}

We simulated the number of infected air travellers intending to fly to a destination country in a given week based on the monthly volume of flights between the origin and destination, and considering the prevalence of COVID-19 in the origin country (Supplementary Table S1). We used the UK as a case study for the destination country. We assumed that the inbound and outbound travel is balanced on average. To estimate the number of people travelling into the UK, we halved the total number of monthly traveller movements.

The time of each intending traveller's flight was sampled uniformly between the time of exposure to SARSCoV-2 and time of recovery. We modelled international travellers coming either from the US or the EU, using publicly available Civil Aviation Authority data for April and May $2020[11,12]$. Estimates of current COVID-19 infection prevalence were derived from reported cases and death time series data while adjusting for reporting delays and under-reporting based on case-fatality ratio estimates $[13,14]$. EU-wide prevalence was calculated as a population-weighted mean of available country-level estimates of the non-UK EU countries (except Malta, for which a prevalence estimate was not available).

For each simulation, we sampled the number of weekly intending travellers, the proportion of those who were infected, and the proportion of infected travellers who were symptomatic and asymptomatic [15] (details are provided in Supplementary Table S1). 


\section{FIGURE 2}

Expected number of infectious and pre-infectious individuals entering the United Kingdom from the United States (A) and total infectious person-days remaining after release (B) based on estimated travel volumes and quarantine duration with no pre-flight testing, United Kingdom, July 2020

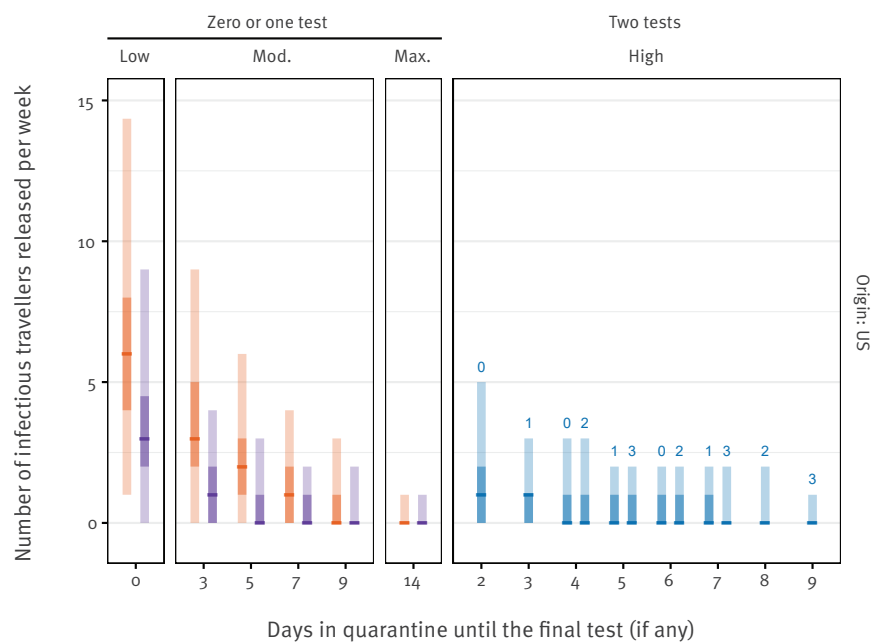

B.

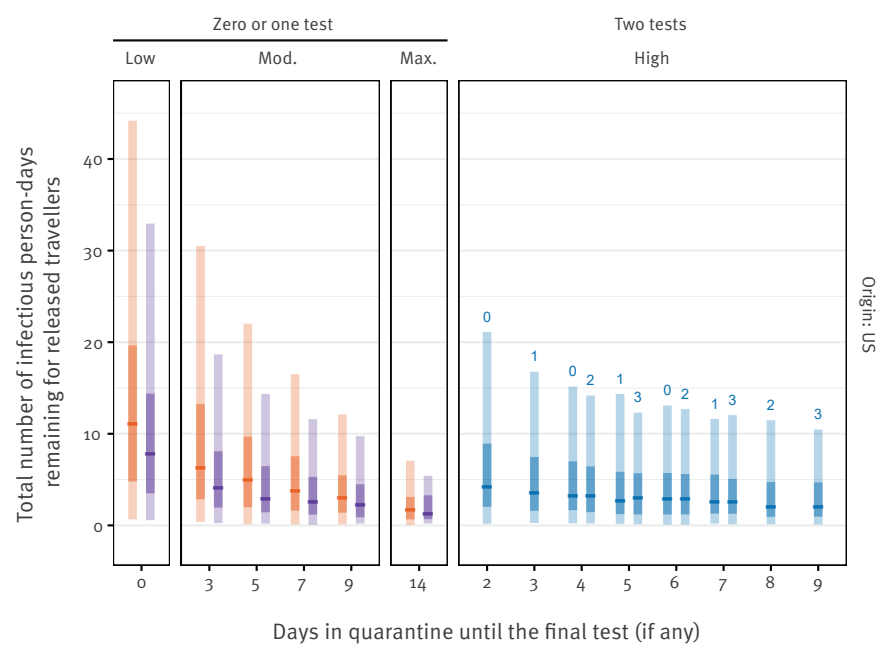

Days until the first of two tests $\quad 0-3$

Number of negative tests required for release $\quad$ Zero $\quad$ One Two

Test results are assumed to be delayed by 1 day and individuals leave quarantine 1 day after their final test. Central dash: median; light bar: $95 \%$ uncertainty interval; dark bar: $50 \%$ uncertainty interval.

\section{Risk mitigation strategies}

We considered several risk mitigation strategies. Travellers are subjected to a quarantine which lasts either (i) o days (low stringency); (ii) 3, 5, 7 or 9 days (moderate stringency); or (iii) 14 days (high/maximum stringency) (Supplementary Table S2). In the low and moderate stringency levels, travellers may also be tested on the final day of their quarantine and wait an additional day for their results [16]; in the low stringency setting, this effectively enforces a 1-day quarantine. For the high stringency scenario, travellers are assumed to undergo two stages of PCR testing; if they receive two negative tests during their post-arrival quarantine period, they are cleared to leave quarantine early (i.e. the day after their final test to account for test delays). Travellers who become symptomatic during their quarantine period must meet all of the following conditions for release: (i) they must no longer display symptoms, (ii) it must be at least 7 days since the onset of symptoms, and (iii) they must have been in quarantine for at least 14 days [3].

\section{Model assumptions}

We assumed that syndromic screening is performed before departure, which may consist of thermal scanning and/or monitoring of symptoms such as cough and fever [17]. Given the awareness of the pandemic and guidance issued on travelling while ill, we assumed in all scenarios that $70 \%$ of currently-symptomatic travellers do not fly (as modelled by Gostic et al. $[18,19]$ ).
Pre-flight PCR testing was required by some countries and airlines. In July 2020, the International Air Transport Association recommended testing within 24 $\mathrm{h}$ of departure [20] but some countries required testing within 7 days of the flight [21]. Considering this wide range of pre-travel test recommendations, we chose to include a 4-day pre-flight test as a midpoint.

\section{Case definitions and detection of infected travellers}

We defined a symptomatic infection as an individual whose symptoms e.g. fever, cough, loss of sense of taste or smell, would be detectable by the individual, airport staff, quarantine staff, or a healthcare worker and typically lead to self-isolation, consistent with that defined by the UK's National Health Service [22]. We defined an asymptomatic infection as one where the individual never develops symptoms throughout the duration of their infection, according to Buitrago-Garcia et al. [15]. We assumed that the sensitivity of PCR testing for a nasopharyngeal or throat swab varies over the course of infection, peaking around onset of symptoms [23], and that test specificity is 100\% [24]. We assumed that the probability of detecting an asymptomatic infection through PCR testing is 0.62 times that of a symptomatic infection, as reported by Chau et al. [25] for nasopharyngeal or throat swab samples collected from quarantining travellers (Supplementary Table S3). We derived the proportion of asymptomatic travellers by quantile, matching the $95 \%$ prediction interval (0.03-0.55) of Buitrago-Garcia et al. as a beta 


\section{FIGURE 3}

Expected number of infectious and pre-infectious individuals entering the United Kingdom from the United States (A) and total infectious person-days remaining after release (B) based on estimated travel volumes and quarantine duration with no pre-flight testing, stratified by asymptomatic or pre-symptomatic infection, United Kingdom, July 2020

A.

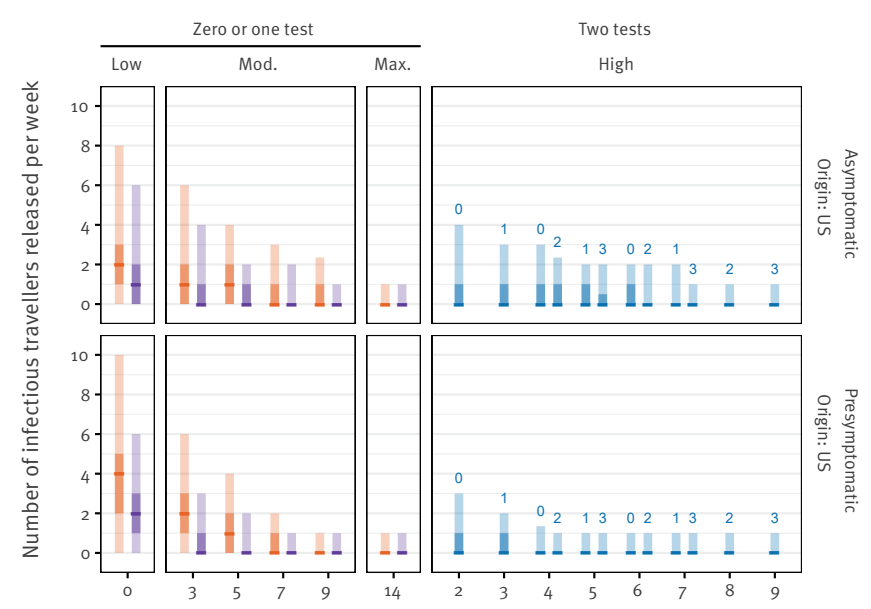

Days in quarantine until the final test (if any)
B.

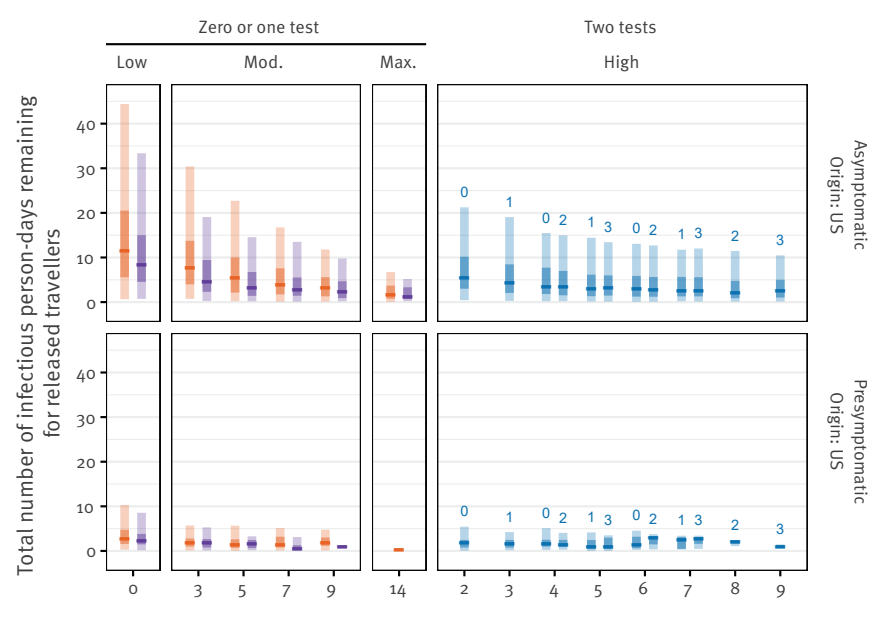

Days in quarantine until the final test (if any)

\section{Days until the first of two tests $\quad 0-3$ \\ Number of negative tests required for release \# Zero \# One \#Two}

Max: maximum; Mod: moderate; US: United States.

Test results are assumed to be delayed by 1 day and individuals leave quarantine 1 day after their final test. Central dash: median; light bar: $95 \%$ uncertainty interval; dark bar: $50 \%$ uncertainty interval.

distribution, giving a median of 0.21 (i.e. $21 \%$ of travellers being asymptomatic on average across model simulations) [15].

The duration of the incubation period (time from exposure to onset of symptoms, and assumed here to also represent the timing of peak probability of detection in both symptomatic and asymptomatic individuals) was taken from Lauer et al. [26] (Supplementary Table S3). The duration of the latent period (time from exposure to the onset of infectiousness) [27], was derived from Ashcroft et al. (corrected version of $\mathrm{He}$ et al.), and was also assumed to be equal for symptomatic and asymptomatic individuals $[28,29]$. The duration of the infectious period of symptomatic cases was derived from Wolfel et al. [30], while that of asymptomatic cases was derived from Byrne et al. [31], with asymptomatic cases being infectious for a shorter period than symptomatic cases (median: 5.2 vs 7.1 days) (Supplementary Table S3).

Given the natural history of infection parameters, we estimated the number of infected travellers entering the community in each scenario who would have the potential to cause onward transmission, i.e. those still in their infectious or pre-infectious period. In addition, we calculated the number of infectious days spent in the community for each infected traveller following their release. These values were then summed for all individuals to give the total person-days of infectiousness spent in the community for each scenario. We report these values for the estimated weekly travellers based on travel volumes and per 10,000 infected travellers, with 1,000 bootstrap replications each to generate medians and $95 \%$ and $50 \%$ uncertainty intervals (UI). We calculated rate ratios (RR) in each screening scenario for the number of infectious individuals released and infectious days remaining compared to the low scenario (syndromic screening, no quarantine, and no PCR testing) and maximum scenario (14-day quarantine, no testing) were calculated with 10,000 travellers per simulation to avoid small number biases and were bootstrapped 1,000 times to generate medians and $95 \%$ and $50 \% \mathrm{UI}$. All analysis was conducted in R version 4.0.2 [32] and the code is available https:// github.com/cmmid/pcr_entry_screening_eurosurv.

\section{Results}

Based on the prevalence of COVID-19 in the respective countries on 20 July 2020 , we estimated that the expected proportion of travellers who entered the UK while infectious was substantially higher for flights originating in the US than for those originating in the EU (Supplementary Figure S3). However, as the prevalence 
Risk reduction per infected traveller compared to a baseline of syndromic screening and no quarantine and no testing on arrival, United Kingdom, July 2020

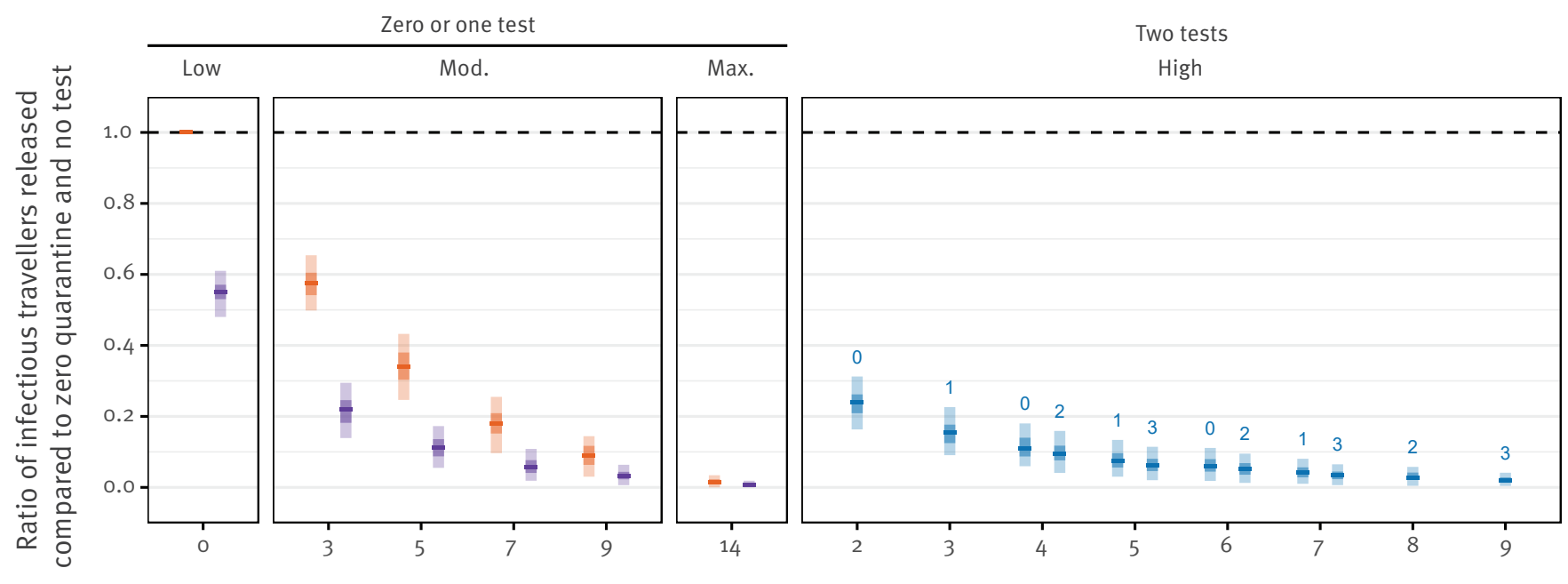

Days in quarantine until the final test (if any)

Days until the first of two tests $0-3$

Number of negative tests required for release - Zero $\quad$ One

Max: maximum; Mod: moderate.

Baseline scenario is indicated by the left-most value (Low, o days quarantine, no testing). We assumed that test results are delayed by 1 day and individuals leave quarantine 1 day after their final test. Central dash: median; light bar: $95 \%$ uncertainty interval; dark bar: $50 \%$ uncertainty interval. Product of 1,000 infected arriving travellers and 1,000 simulations per scenario.

of COVID-19 in the US was ca 14 times that in the EU in July 2020 and travel volumes were ca 8 times lower than those from the EU, we expect approximately half the number of infectious travellers arriving from the EU than from the US (Supplementary Table S1). Here we focus on the estimates for travel from the US and provide results for travel from the EU for comparison in the Supplement (Figure S1 and S2).

\section{Effectiveness of quarantine and testing}

As a baseline for comparison, we used the lowest stringency scenario considered i.e. $70 \%$ of currently symptomatic travellers are prevented from boarding, but no quarantine or testing is conducted. In this scenario, a median of six infectious travellers (95\% UI: 1-14.2) would enter the community from the US per week (Figure 2A). By introducing a mandatory quarantine period of 7 days, this can be reduced to one infectious traveller (95\% UI: $0-4$ ), preventing ca $80 \%$ of infectious travellers from entering the community (RR: 0.17; 95\% UI: 0.10-0.26). A mandatory quarantine period of 14 days resulted in zero to one infectious entry per week, almost fully preventing importation (RR: 0.02; 95\% Ul: 0.00-0.03).
Longer quarantine periods increase the fraction of presymptomatic infected travellers who would have their onset of symptoms during the quarantine and hence self-isolate until symptoms subside (Figure 2A, Figure $3 A)$. Accordingly, we estimated a more pronounced impact of interventions targeting travellers on the number of infectious person-days from travellers, particularly for those who would eventually become symptomatic (Figure 2B, Figure $3 \mathrm{~B}$ ). The uncertainty in the number of remaining infectious person-days is driven by variability in the detection of asymptomatic infections, as they will never be detected by pre-flight syndromic screening, are less likely to be detected by PCR and will never develop symptoms that trigger mandatory isolation.

Conducting a single test for all travellers at the end of the described quarantine periods further reduced the median number of infectious entering travellers from the US, with an RR of 0.55 (95\% UI: 0.48-0.61) for a test on arrival, with release on day 1 ; an RR of 0.11 (95\% UI: $0.05-0.17$ ) for a test on day 5 , release on day 6; an RR of 0.06 (95\% UI: $0.02-0.11$ ) for a test on day 7, release on day 8; an RR of 0.03 (95\% UI: $0.00-0.06$ ) for a test on day 9 with release on day 10; and an RR of 0.01 (95\% UI: $0.00-0.02$ ) for a test on day 14 , release 
Risk reduction per infected traveller compared to a baseline of syndromic screening and no quarantine and no testing on arrival, stratified by asymptomatic or pre-symptomatic infection, United Kingdom, July 2020

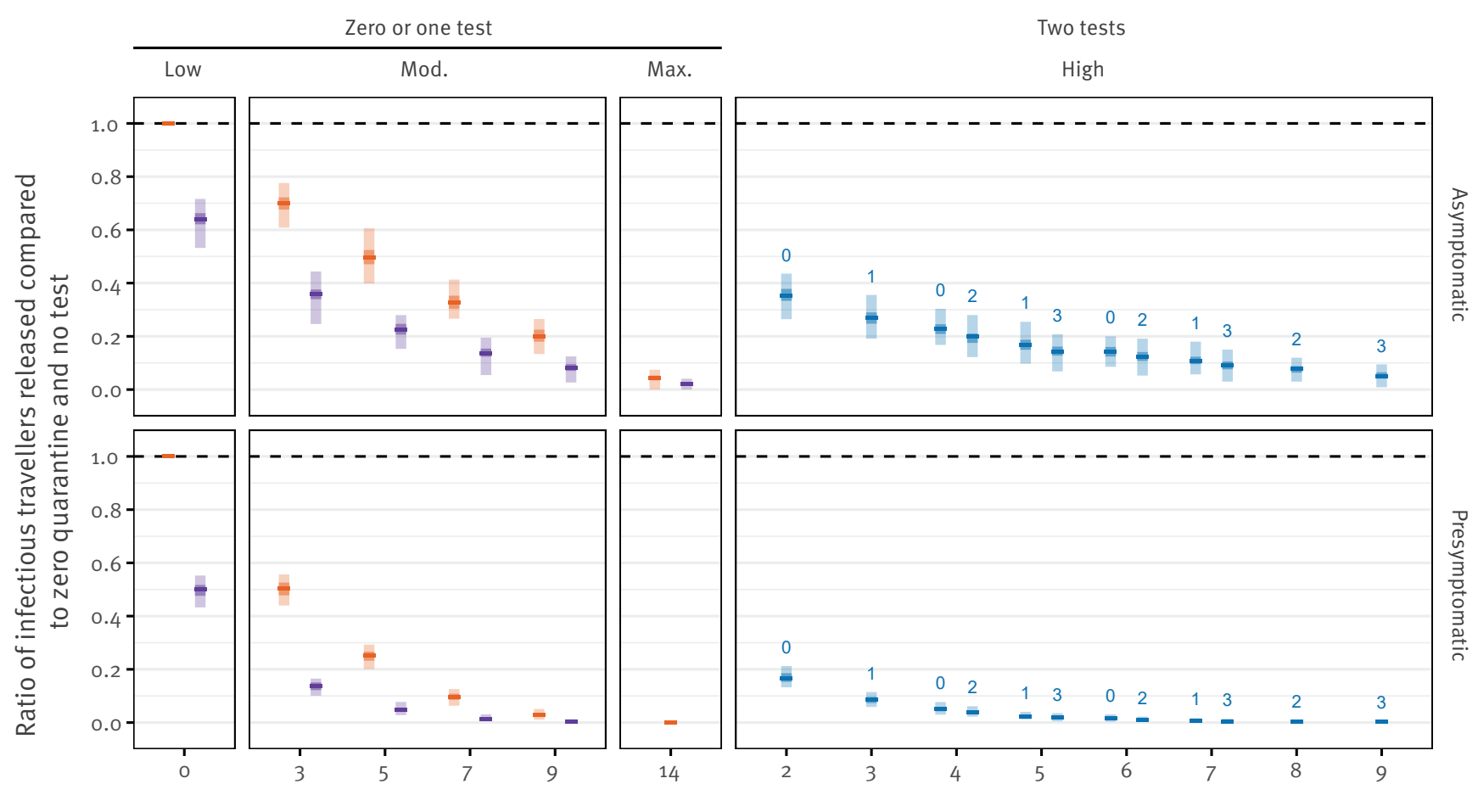

Days in quarantine until the final test (if any)

Days until the first of two tests $0-3$

Number of negative tests required for release $=$ Zero $\quad$ One Two

Max: maximum; Mod: moderate.

Baseline scenario is indicated by the left-most value (Low, o days quarantine, no testing). We assumed that test results are delayed by 1 day and individuals leave quarantine 1 day after their final test. Central dash: median; light bar: $95 \%$ uncertainty interval; dark bar: $50 \%$ uncertainty interval. Product of 1,000 infected arriving travellers and 1,000 simulations per scenario.

on day 15 , when compared with the lowest stringency scenario (Figure 4). Requiring a second round of testing had marginal impact, although a quarantine period of 9 days with two tests and early exit may be able to largely replicate the impact of a 14 -day quarantine period (RR: 0.02; 95\% UI: 0.00-0.04).

\section{Rate ratios by symptom status}

We stratified the above RR by whether the infection is asymptomatic or pre-symptomatic. We observed that the strategies are more effective against those with pre-symptomatic than asymptomatic infections (Figure 5). The introduction of a test on arrival (Figure 5, low, 1 day) reduces the number of asymptomatic entering travellers by $36 \%$ (95\% UI: 28-47) and pre-symptomatic by $50 \%$ ( $95 \%$ UI: $45-56$ ), beyond that which is captured by syndromic screening alone (Figure 5, low, o days). At maximum stringency, a 14-day quarantine period is able to reduce the number of symptomatic entering travellers by more than $99 \%$ (95\% UI: 99-100) and asymptomatic entering travellers by $96 \%$ (95\% UI: 93-100).

For single test strategies, a 9-day quarantine with no test reduces the symptomatic entering travellers by 97\% (95\% UI: 95-99) but asymptomatic entering travellers are only reduced by $80 \%$ (95\% UI: 4-87), reflecting the difficulty of relying on symptom onset during quarantine. By introducing a PCR test on day 9 and release on day 10 , the number of symptomatic entering travellers is reduced by more than $99 \%$ (95\% UI: 9-100) and asymptomatic entering travellers by $92 \%$ (95\% UI: 88-97). This difference in detectability of symptomatic and asymptomatic entering travellers, coupled with simulations involving their small absolute numbers, is responsible for driving the wide uncertainty observed in the number of infectious arriving travellers from the US entering the community (Figure 2). 
Effect of reducing the duration of quarantine

To determine if a 14-day quarantine can be replaced by a shorter quarantine with testing, we made RR comparisons to a 14-day quarantine with no test. We see that shorter quarantine periods of 9 days with either one or two rounds of testing may have a similar effect to that of the 14-day quarantine period (RR: 2.0 ; $95 \%$ UI: $1.00-$ infinity for a test on day 9 with release on day 10; RR: 1.24; $95 \%$ UI: 0.53 -infinity for a test on day 3 and day 9) (Supplementary Figure S6).

\section{Pre-flight testing}

The impact of pre-flight testing on the number of infectious travellers entering the community was greatest if implemented the day before departure (within $24 \mathrm{~h}$ ) in scenarios with no post-flight testing, with an RR of 0.69 (95\% UI: 0.64-0.73) compared with no testing either before departure or after arrival (Supplementary Figure S4). As quarantine increases in duration, the additional effect of pre-flight testing diminishes.

\section{Discussion}

Here we analysed the effect of different combinations of PCR testing and quarantine times on the number of infectious individuals entering a country, beyond the effect of syndromic screening at the site of departure in the pre-COVID-19 vaccination era. We found that a quarantine period of at least 5 days, combined with a single PCR test on the final day, resulted in a reduction of $89 \%$ in the number of infectious individuals entering the community. A 7-day quarantine with a test on the final day can reduce infectious entering travellers by an average of $95 \%$, with a small marginal benefit for additional rounds of testing. In addition, pre-flight testing appears ineffective unless conducted within 24 $\mathrm{h}$ of departure; the marginal effect of these tests disappears with increased quarantine duration and postarrival testing.

We also found that the 14-day quarantine period is highly effective, reducing the number of infectious entering travellers by $99 \%$, on average. Because the 14-day quarantine strategy almost completely eliminates infectious entering travellers, the number of infected entering travellers for other strategies (i.e. 7 or 9 days of quarantine with testing) may represent a twoto fivefold increase in RR. However, the absolute risk of entry is small in these scenarios and so the increase in RR should be interpreted in light of this. The risk stemming from the arrival of infectious travellers will need to be assessed in the context of local infection incidence. For example, six to 11 infectious travellers arriving per week from the EU or US in July 2020 into a community with thousands of live infections (such as that in the UK on 12 June 2020 , with a prevalence estimated at 45 infections per 10,000 inhabitants; 95\% UI: 24-92) will likely have little impact on control efforts. In contrast, if local infection prevalence is lower (as in the UK on 20 July 2020 with an estimated nine infections per 10,000 inhabitants; 95\% UI: 4-18) a higher number of incoming infectious travellers may pose a large risk for seeding outbreaks in the community [33]. Likewise, countries that have pursued policies to eliminate COVID-19 within their borders such as New Zealand may consider any risk of reintroduction as unacceptable $[2,34]$ and therefore continue to pursue policies which minimise the risk as much as practically possible.

We presented the risk from incoming infected travellers as the number of infectious travellers entering the community. To account for the differential residual duration of their infectiousness with the different strategies, we also presented the number of infectious persondays in the community from travellers. While the latter measure indicates an increased effectiveness of longer quarantine, this approach may still underestimate the true effect since the measure only considers that travellers are still infectious, and not how likely transmission is given the viral load. Of note, it is likely that infectiousness is correlated with viral load and declines over the course of infection [35,36]. Hence, with a peak infectivity around the onset of symptoms at ca 5 days, a minimum quarantine period of 7 days is likely to result in the release of fewer infectious travellers, and those who are released at 7 days have less potential for transmission with or without the use of a test.

We assumed that inbound travel volumes were $50 \%$ of total traveller movements, as reported by the Civil Aviation Authority, which may not reflect asymmetric patterns of travel. The total number of traveller movements between the UK and both the US and the EU in April and May 2020 were ca $1 \%$ of that reported in 2019, indicating that the combination of travel restrictions and suspended airline flights led to a sharp decrease in the number of potentially infected travellers. If travel volumes return to pre-pandemic levels, the likely number of infectious arriving travellers will increase unless prevalence is severely reduced internationally or a greater proportion of the international population is either vaccinated or naturally infected and recovered. In our analysis, we considered a constant air passenger volume and did not consider that shortening of quarantine may lead to an increase in the number of travellers. To address this, we have provided estimates in terms of the number of infectious entering travellers per 10,000 arriving travellers for the given international prevalence.

The work presented here is based on estimates of prevalence, under-ascertainment and travel volumes as of July 2020, as well as the contemporaneous understanding of incubation period, infectiousness and ability to detect an infection by PCR. Multiple studies have found that infectivity peaks around onset of symptoms $[28,35,36]$ and that asymptomatic infections follow similar peak timings but that the detectability by PCR is shorter in duration [37] and lesser in magnitude [25]. We assumed that $70 \%$ of travellers with currently symptomatic infections (e.g. with a cough or fever) 
would be detected or self-report and hence not travel. The remaining 30\% are therefore the infectious travellers, who are either symptomatic but undetected, presymptomatic or fully asymptomatic. The longer the duration of quarantine, the greater the chance that pre-symptomatic individuals will develop symptoms and self-isolate, further reducing the number of infectious entering travellers. Hence, the primary purpose of quarantine and PCR testing is to reduce possible transmission from asymptomatic travellers who are only detectable by PCR. We also assumed that if individuals subsequently become symptomatic after quarantine, they follow national guidelines to immediately re-enter quarantine and seek an additional test as part of the local test and trace strategy; we assumed that traveller sensitisation is high at this point in the pandemic $[38,39]$. We do not make any assumptions about the potential for self-isolating infectious travellers to infect their household upon arrival or the resulting onwards transmission. We assumed full adherence to self-isolation, although a first negative test and a long duration of quarantine may reduce adherence to quarantine rules $[6,8]$. Hence, by assuming perfect adherence, we may overestimate the added benefit of long periods of quarantine in terms of the person-days of infectiousness in the community.

\section{Conclusions}

As the pandemic progresses, public health authorities must carefully balance the need for travellertargeted interventions that reduce the likelihood of seeding local COVID-19 outbreaks with their social, psychological, financial, and economic costs. While the acceptable number of infected travellers entering the community will depend on the local context of SARS-CoV-2 transmission, we found that for travellers arriving from low prevalence destinations, the absolute risk of infectious entering travellers is likely to be low. Hence, testing and/or quarantine-based strategies may not reduce such risk further, particularly when many infectious arriving travellers are asymptomatic. However, as we have highlighted here, testing is likely the only way to detect asymptomatic infections, and may also detect pre-symptomatic, infectious travellers, leading to earlier isolation. For arriving travellers from countries with ongoing community transmission, quarantine on arrival will limit the risk for onward transmission into the local community in the absence of a safe and effective vaccine against COVID-19. While a 14-day quarantine will likely prevent most transmission from travellers, an 8-day quarantine (with testing on day 7) can capture almost as many infectious individuals in approximately half the time. Testing passengers is resource-intensive but presents a way to either further reduce risks or allow a shorter quarantine at the same level of risk, particularly for travellers arriving from countries with widespread SARS-CoV-2 transmission. Thus, our results contribute to an evidence-based discussion on the benefits and risks of alternative policies on border security regarding SARS-CoV-2 introduction via international air travel.
Investigators

Centre for Mathematical Modelling of Infectious Diseases (CMMID) COVID-19 Working Group: Katharine Sherratt, Stéphane Hué, Matthew Quaife, Nikos I Bosse, Graham Medley, Megan Auzenbergs, Adam J Kucharski, Nicholas G Davies, Oliver Brady, Sophie R Meakin, Rein M G J Houben, Katherine E Atkins, Kiesha Prem, C Julian Villabona-Arenas, Hamish P Gibbs, Thibaut Jombart, Charlie Diamond, Petra Klepac, Arminder K Deol, Rachel Lowe, James W Rudge, Mark Jit, Sebastian Funk, Gwenan M. Knight, Simon R. Procter, David Simons, Quentin J Leclerc, James D Munday, Amy Gimma, Georgia R Gore-Langton, Christopher I Jarvis, Jon C Emery, Anna M Foss, Kathleen O'Reilly, Joel Hellewell, Emily S Nightingale, Kevin van Zandvoort, Damien C Tully, Sam Abbott, Kaja Abbas, Fiona Yueqian Sun, Alicia Rosello

\section{Acknowledgements}

Funding: The following funding sources are acknowledged as providing funding for the named authors. This research was partly funded by the Bill \& Melinda Gates Foundation (INV003174: YL; NTD Modelling Consortium OPP1184344: (ABP). DFID/Wellcome Trust (Epidemic Preparedness Coronavirus research programme $221303 / \mathrm{Z} / 20 / \mathrm{Z}$ : (ABP). This project has received funding from the European Union's Horizon 2020 research and innovation programme - project EpiPose (101003688: WJE, YL). HDR UK (MR/So03975/1: RME). This research was partly funded by the National Institute for Health Research (NIHR) using UK aid from the UK Government to support global health research. The views expressed in this publication are those of the author(s) and not necessarily those of the NIHR or the UK Department of Health and Social Care (16/136/46: BJQ; 16/137/109: BJQ, YL; PR-OD-1017-20002: WJE). UK MRC (MC_PC_19065 - Covid 19: Understanding the dynamics and drivers of the COVID-19 epidemic using realtime outbreak analytics: RME, SC, WJE, YL). Wellcome Trust (206250/Z/17/Z: TWR; 208812/Z/17/Z: SC, SFlasche). The Alan Turing Institute and The Nakajima Foundation (AE). No funding (YWDC).

The following funding sources are acknowledged as providing funding for the working group authors. BBSRC LIDP (BB/Mo09513/1: DS). This research was partly funded by the Bill \& Melinda Gates Foundation (INV-001754: MQ; INV003174: KP, MJ; NTD Modelling Consortium OPP1184344: GM; OPP1180644: SRP; OPP1183986: ESN; OPP1191821: KO'R, MA). BMGF (OPP1157270: KA). DFID/Wellcome Trust (Epidemic Preparedness Coronavirus research programme 221303/Z/20/Z: KvZ). DTRA (HDTRA1-18-1-0051: JWR). Elrha R2HC/UK DFID/Wellcome Trust/This research was partly funded by the National Institute for Health Research (NIHR) using UK aid from the UK Government to support global health research. The views expressed in this publication are those of the author(s) and not necessarily those of the NIHR or the UK Department of Health and Social Care (KvZ). ERC Starting Grant (\#757688: CJVA, KEA; \#757699: JCE, $M Q, R M G J H)$. This project has received funding from the European Union's Horizon 2020 research and innovation programme - project EpiPose (101003688: KP, MJ, PK). This research was partly funded by the Global Challenges Research Fund (GCRF) project 'RECAP' managed through RCUK and ESRC (ES/P010873/1: AG, CIJ, TJ). NIHR (16/137/109: CD, FYS, MJ; Health Protection Research Unit for Immunisation NIHR200929: NGD; Health Protection Research Unit for Modelling Methodology HPRU-2012-10096: TJ; NIHR200929: MJ; PR-OD-1017-20002: AR). Royal Society (Dorothy Hodgkin Fellowship: RL; RP\EA\180004: PK). UK DHSC/UK Aid/NIHR (ITCRZ 03010: HPG). UK MRC (LID DTP MR/N013638/1: GRGL, QJL; MC_PC_19065 - Covid 19: Understanding the dynamics and drivers of the COVID-19 epidemic using real-time outbreak analytics: AG, NGD, TJ; MR/P014658/1: GMK). Authors of this research receive funding from UK Public Health Rapid 
Support Team funded by the United Kingdom Department of Health and Social Care (TJ). Wellcome Trust (206250/Z/17/Z: AJK; 206471/Z/17/Z: OJB; 210758/Z/18/Z: JDM, JH, KS, NIB, SA, SFunk, SRM). No funding (AKD, AMF, DCT, SH).

\section{Conflict of interest}

Akira Endo received a research grant from Taisho Pharmaceutical Co. Ltd.

\section{Authors' contributions}

SC, BJQ, SF and WJE conceived the study and wrote the report. SC, BJQ, AE, SF and WJE designed the model. SC and $B J Q$ led the development and analysis of the screening model and produced the results and figures. TWR led the development of and analysis of the prevalence model. TWR, YL, YWDC, CABP, AE, SF, RME and WJE consulted on the analyses. The CMMID COVID-19 Working Group members contributed by interpreting the study findings, contributing to the report, and approving the work for publication. All authors approved the final version for publication.

\section{References}

1. Chinazzi M, Davis JT, Ajelli M, Gioannini C, Litvinova M, Merler S, et al. The effect of travel restrictions on the spread of the 2019 novel coronavirus (COVID-19) outbreak. Science. 2020;368(6489):395-400. https://doi.org/10.1126/science. aba9757 PMID: 32144116

2. Han E, Tan MMJ, Turk E, Sridhar D, Leung GM, Shibuya K, et al. Lessons learnt from easing COVID-19 restrictions: an analysis of countries and regions in Asia Pacific and Europe. Lancet. 2020;396(10261):1525-34. https://doi.org/10.1016/S01406736(20)32007-9 PMID: 32979936

3. Department of Health and Social Care and Department for Transport. Coronavirus (COVID-19): how to self-isolate when you travel to the UK. London: United Kingdom Government. [Accessed: 20 Jul 2020]. Available from: https://www.gov.uk/ government/publications/coronavirus-covid-19-how-to-selfisolate-when-you-travel-to-the-uk/coronavirus-covid-19-howto-self-isolate-when-you-travel-to-the-uk

4. Li Q, Guan X, Wu P, Wang X, Zhou L, Tong Y, et al. Early transmission dynamics in Wuhan, China, of novel coronavirusinfected pneumonia. N Engl J Med. 2020;382(13):1199-207. https://doi.org/10.1056/NEJMoa2001316 PMID: 31995857

5. World Health Organisation (WHO). Operational considerations for case management of COVID-19 in health facility and community. Report No.: WHO/2019-nCoV/ HCF_operations/2020.1. Geneva: WHO; 2020. Available from: https://www.who.int/publications/i/item/10665-331492

6. Brooks SK, Webster RK, Smith LE, Woodland L, Wessely S, Greenberg N, et al. The psychological impact of quarantine and how to reduce it: rapid review of the evidence. Lancet. 2020;395(10227):912-20. https://doi.org/10.1016/S01406736(20)30460-8 PMID: 32112714

7. Pfefferbaum B, North CS. Mental health and the covid-19 pandemic. N Engl J Med. 2020;383(6):510-2. https://doi. org/10.1056/NEJMp2008017 PMID: 32283003

8. Webster RK, Brooks SK, Smith LE, Woodland L, Wessely S Rubin GJ. How to improve adherence with quarantine: rapid review of the evidence. Public Health. 2020;182:163-9. https:// doi.org/10.1016/j.puhe.2020.03.007 PMID: 32334182

9. United States Food and Drug Administration (FDA). Quest SARS-CoV-2 rRT-PCR (Quest Diagnostics Infectious Disease, Inc.) - Manufacturer Instructions/Package Insert. Silver Spring: FDA. [Accessed: 16 Jul 2020]. Available from: https://www.fda. gov/media/136231/

10. Nikkei Staff Writers. Japan set to lift quarantines for business travelers in summer. Nikkei Asian Review. [Accessed: 6 Jul 2020]. Available from: https://asia.nikkei.com/Business/Travel-Leisure/ Japan-set-to-lift-quarantines-for-business-travelers-in-summer

11. Civil Aviation Authority. Airport data. 201907 | London: Civil Aviation Authority. [Accessed: 20 Jul 2020]. Available from: https://www.caa.co.uk/Data-and-analysis/UK-aviation-market/ Airports/Datasets/UK-Airport-data/Airport-data-2019-07
12. Civil Aviation Authority. Airport data. 2020 05. London: Civil Aviation Authority. [Accessed: 4 Jul 2020]. Available from: https://www.caa.co.uk/Data-and-analysis/UK-aviation-market/ Airports/Datasets/UK-Airport-data/Airport-data-2020-05

13. Russell TW, Golding N, Hellewell J, Abbott S, Wright L, Pearson $\mathrm{CAB}$, et al. Reconstructing the early global dynamics of under-ascertained COVID-19 cases and infections. BMC Med. 2020;18(1):332. https://doi.org/10.1186/s12916-020-01790-9 PMID: 33087179

14. Russell TW, Wu JT, Clifford S, Edmunds WJ, Kucharski AJ, Jit $M$, et al. Effect of internationally imported cases on internal spread of COVID-19: a mathematical modelling study. Lancet Public Health. 2021;6(1):e12-20. https://doi.org/10.1016/ S2468-2667(20)30263-2 PMID: 33301722

15. Buitrago-Garcia D, Egli-Gany D, Counotte MJ, Hossmann $\mathrm{S}$, Imeri H, Ipekci AM, et al. Occurrence and transmission potential of asymptomatic and presymptomatic SARS-CoV-2 infections: A living systematic review and meta-analysis. PLoS Med. 2020;17(9):e1003346. https://doi.org/10.1371/journal. pmed.1003346 PMID: 32960881

16. United Kingdom Government. Coronavirus (COVID-19): getting tested. London: gov.uk. [Accessed: 12 Jun 2020]. Available from: https://www.gov.uk/guidance/ coronavirus-covid-19-getting-tested

17. Quilty BJ, Clifford S, Flasche S, Eggo RM, CMMID nCoV working group. Effectiveness of airport screening at detecting travellers infected with novel coronavirus (2019-nCoV). Euro Surveill. 2020;25(5):2000080. https://doi.org/10.2807/1560-7917. ES.2020.25.5.2000080 PMID: 32046816

18. Gostic K, Gomez AC, Mummah RO, Kucharski AJ, Lloyd-Smith JO. Estimated effectiveness of symptom and risk screening to prevent the spread of COVID-19. eLife. 2020;9:e55570. https:// doi.org/10.7554/eLife.55570 PMID: 32091395

19. Gostic K. Estimated effectiveness of symptom and risk screening to prevent the spread of COVID-19. San Francisco: Github. [Accessed: 16 Jul 2020]. Available from: https:// kgostic.github.io/traveller_screening

20. International Air Transport Association (IATA). Criteria for COVID-19 testing in the air travel process. Geneva: IATA. [Accessed: 4 Jul 2020]. Available from: https://www.iata.org/ en/pressroom/pr/2020-06-16-02/

21. Xinhua Global Service. [Beijing Railway Department: Implementing ticket purchase restrictions for high-risk groups and leaving Beijing with a negative certificate of nucleic acid test within 7 days]. Xinhuanet. [Accessed: 6 Jul 2020]. Chinese. Available from: http://www.xinhuanet.com/politics/202006/23/c_1126152337.htm

22. National Health Service (NHS). Main symptoms of coronavirus (COVID-19). London: NHS. [Accessed: 20 Jul 2020]. Available from: https://www.nhs.uk/conditions/coronavirus-covid-19/ symptoms/main-symptoms

23. Kucirka LM, Lauer SA, Laeyendecker O, Boon D, Lessler J. Variation in false-negative rate of reverse transcriptase polymerase chain reaction-based SARS-CoV-2 tests by time since exposure. Ann Intern Med. 2020;173(4):262-7. https:// doi.org/10.7326/M20-1495 PMID: 32422057

24. Grassly N, Pons Salort M, Parker E, White P, Ainslie K, Baguelin $M$, et al. Report 16: Role of testing in COVID-19 control. London: Imperial College London; 2020. Available from: https://spiral. imperial.ac.uk:8443/handle/10044/1/78439

25. Van Vinh Chau N, Lam VT, Dung NT, Yen LM, Minh NNQ, Hung $L M$, et al. The natural history and transmission potential of asymptomatic SARS-CoV-2 infection. Clin Infect Dis. 2020;71(10):2679. https://doi.org/10.1093/cid/ciaa711 PMID: 32497212

26. Lauer SA, Grantz KH, Bi Q, Jones FK, Zheng Q, Meredith HR, et al. The incubation period of coronavirus disease 2019 (COVID-19) from publicly reported confirmed cases: estimation and application. Ann Intern Med. 2020;172(9):577-82. https:// doi.org/10.7326/M20-0504 PMID: 32150748

27. Davies NG, Klepac P, Liu Y, Prem K, Jit M, CMMID COVID-19 working group, Eggo RM. Age-dependent effects in the transmission and control of COVID-19 epidemics. Nat Med. 2020;26(8):1205-11. https://doi.org/10.1038/s41591-0200962-9 PMID: 32546824

28. He X, Lau EHY, Wu P, Deng X, Wang J, Hao X, et al. Temporal dynamics in viral shedding and transmissibility of COVID-19. Nat Med. 2020;26(5):672-5. https://doi.org/10.1038/s41591020-0869-5 PMID: 32296168

29. Ashcroft P, Huisman JS, Lehtinen S, Bouman JA, Althaus CL, Regoes RR, et al. COVID-19 infectivity profile correction. Swiss Med Wkly. 2020;150:w20336. PMID: 32757177

30. Wölfel R, Corman VM, Guggemos W, Seilmaier M, Zange S Müller MA, et al. Virological assessment of hospitalized patients with COVID-2019. Nature. 2020;581(7809):465-9. https://doi.org/10.1038/S41586-020-2196-x PMID: 32235945 
31. Byrne AW, McEvoy D, Collins AB, Hunt K, Casey M, Barber A, et al. Inferred duration of infectious period of SARS-CoV-2: rapid scoping review and analysis of available evidence for asymptomatic and symptomatic COVID-19 cases. BM Open. 2020;10(8):e039856. https://doi.org/10.1136/ bmjopen-2020-039856 PMID: 32759252

32. R Core Team. R: A Language and Environment for Statistical Computing. Vienna: R Foundation for Statistical Computing; 2020. Available from: https://www.R-project.org

33. Russell TW, Wu JT, Clifford S, Edmunds WJ, Kucharski AJ, Jit M, Centre for the Mathematical Modelling of Infectious Diseases COVID-19 working group. Effect of internationally imported cases on internal spread of COVID-19: a mathematica modelling study. Lancet Public Health. 2021;6(1):e12-20. https://doi.org/10.1016/S2468-2667(20)30263-2 PMID: 33301722

34. Baker MG, Kvalsvig A, Verrall AJ. New Zealand's COVID-19 elimination strategy. Med J Aust. 2020;213(5):198-200.e1. https://doi.org/10.5694/mja2.50735 PMID: 32789868

35. Cevik M, Tate M, Lloyd O, Maraolo AE, Schafers J, Ho A. SARSCoV-2, SARS-CoV, and MERS-CoV viral load dynamics, duration of viral shedding, and infectiousness: a systematic review and meta-analysis. Lancet Microbe. 2021;2(1):e13-22. https://doi. org/10.1016/S2666-5247(20)30172-5 PMID: 33521734

36. Kissler SM, Fauver JR, Mack C, Olesen SW, Tai C, Shiue KY, et al. Viral dynamics of acute SARS-CoV-2 infection and applications to diagnostic and public health strategies. PLoS Biol. 2021;19(7):e3001333. https://doi.org/10.1371/journal. pbio.3001333 PMID: 34252080

37. Quilty BJ, Clifford S, Hellewell J, Russell TW, Kucharski AJ, Flasche $S$, et al. Quarantine and testing strategies in contact tracing for SARS-CoV-2: a modelling study. Lancet Public Health. 2021;6(3):e175-83. https://doi.org/10.1016/S24682667(20)30308-X PMID: 33484644

38. Hellewell J, Abbott S, Gimma A, Bosse NI, Jarvis Cl, Russell TW, et al. Feasibility of controlling COVID-19 outbreaks by isolation of cases and contacts. Lancet Glob Health. $2020 ; 8(4): \mathrm{e} 488$ 96. https://doi.org/10.1016/S2214-109X(20)30074-7 PMID: 32119825

39. Clifford S, Pearson CAB, Klepac P, Van Zandvoort K, Quilty BJ, Eggo RM, et al. Effectiveness of interventions targeting air travellers for delaying local outbreaks of SARS-CoV-2. Travel Med. 2020;27(5):taaa068. https://doi.org/10.1093/jtm/ taaao68 PMID: 32384159

40. Pya N, Wood SN. Shape constrained additive models. Stat Comput. 2015;25(3):543-59. https://doi.org/10.1007/ S11222-013-9448-7

41. Devroye L. General Principles in Random Variate Generation. Non-Uniform Random Variate Generation. Springer: New York, NY; 1986. https://doi.org/10.1007/978-1-4613-8643-8_2

\section{License, supplementary material and copyright}

This is an open-access article distributed under the terms of the Creative Commons Attribution (CC BY 4.0) Licence. You may share and adapt the material, but must give appropriate credit to the source, provide a link to the licence and indicate if changes were made.

Any supplementary material referenced in the article can be found in the online version.

This article is copyright of the authors or their affiliated institutions, 2021. 